\title{
Influence of change in ROS production in plant cells on resistance of agricultural plants to oxidative stress
}

\author{
Olga Zhuikova ${ }^{1}$, Vladislav Yemelyanov ${ }^{2}$, and Olga Bolotnikova ${ }^{1,3, *}$ \\ ${ }^{1}$ Peter the Great St. Petersburg Polytechnic University, 195251, 29 Polytechnicheskaya Str., \\ Saint Petersburg, Russian Federation \\ ${ }^{2}$ St. Petersburg State University, 199034, 7/9 Universitetskaya Emb., Saint Petersburg, \\ Russian Federation \\ ${ }^{3}$ Petrozavodsk State University, 185910, Lenina Str. 33, Petrozavodsk, Republic of Karelia, \\ Russian Federation
}

\begin{abstract}
The paper considers theoretical data on the mechanisms of the formation of reactive oxygen species (ROS) and the transduction of stress signals in plant cells. The negative effects of ROS with excessive generation are described. A method of protoplast isolation and a fluorescent microscopy were selected for the experiments. Also, in this work, a comparative analysis of the production of ROS during hypoxia and reaeration for agricultural plants that are differently resistant to the described stress is carried out. Based on the results of the experimental part of the work, conclusions were drawn and the practical application of this study was described.
\end{abstract}

\section{Introduction}

It is known that in natural conditions, living things are subject to the negative impacts of various environmental factors. Thus, higher plants growing in aquatic, semi-aquatic ecosystems and also in arid areas suffer from low soil oxygen conditions [1]. Long-term evolution ensured the development of physiological, morphological and metabolic adaptations of plant tissues to hypoxic (anoxic) conditions. The most important of them are considered the rapid growth of shoots, the accelerated formation of adventitious roots and aerenchyma [2]. These adaptations stimulate the transport of oxygen to the flooded root. At the same time, they induce a variety of metabolic changes that prevent the hypoenergetic state of cells, intoxication with the intermediate and the end products of anaerobic metabolism, and maintain the cytosolic $\mathrm{pH}$ [3]. Despite a good understanding of the molecular basis of plant adaptation to oxidative stress, the hypoxic (anoxic) signal transduction mechanism in plant cells is not fully understood.

It is widely believed that intracellular "signaling", mediating the adaptation of plants to various unfavourable factors, is realized through the formation of the so-called "stress" metabolites. A special place among them is occupied by reactive oxygen species (ROS) [4,

${ }^{*}$ Corresponding author: olga-bolotnikova@rambler.ru 
5]. It has been established that the level of $\mathrm{H}_{2} \mathrm{O}_{2}$ production is an important element in the development of the plant cell response to the action of various stress factors [6]. Therefore, finding out the level of $\mathrm{H}_{2} \mathrm{O}_{2}$ participation in the activation of defence mechanisms during the oxygen deprivation is relevant and significant for the formulation of selection strategy for new high-yielding plant varieties [7]. Today fluorescent probes are widely used to analyze the signaling systems of plant cells. The use of the fluorescence method and confocal microscopy makes it possible to record even insignificant changes in the concentration of messenger molecules transmitting signals from the external environment [8]. Therefore, the aim of this work was to comparative analyze the $\mathrm{H}_{2} \mathrm{O}_{2}$ content in the protoplasts of spring wheat (Triticum aestivum L.) and rice (Oryza sativa L.) under conditions of anoxia and postanoxic oxidative action using fluorescent probes.

\section{Methods}

To isolate protoplasts, seeds of spring wheat (Triticum aestivum L., cv. Leningradka) and rice (Oryza sativa L., Japanese variety, cv. Raptor) were germinated. Seeds were sterilized with $5 \% \mathrm{NaOCl}$ for 15 minutes. After that, the seeds were thoroughly washed several times and placed in hot water at temperatures of $+45-50^{\circ} \mathrm{C}$ (wheat) and $+50-55^{\circ} \mathrm{C}$ (rice) for 1 hour. Seed germination was carried out in the thermostat for $2-3$ days $\left(+20-25^{\circ} \mathrm{C}\right)$. Then seedlings were placed on a perforated plate so that the roots were immersed in aerated $0.2 \%$ Knop solution. Further cultivation of seedlings was carried out in the plant growth chamber at $+22-28^{\circ} \mathrm{C}$ for $3-4$ days at a relative humidity of at least $95 \%$. To isolate protoplasts, 1 gram of leaves was taken, sliced into $0.5 \mathrm{~mm}$ fragments and placed in the enzymatic solution for $2-3$ hours at $+30^{\circ} \mathrm{C}$. The solution for the isolation of protoplasts included: $0.5 \mathrm{M}$ sorbitol; 0.2\% BSA (Bovine Serum Albumin); 0.05\% PVP (polyvinylpyrrolidone); $1 \mathrm{mM} \mathrm{CaCl} 2 ; 1-2 \%$ Trichoderma reesei cellulase (EC 3.2.1.4., Sigma); 0.3-0.5\% macerase "Maceroenzyme R-10" (EC 3.2.1.4., Serva); 20 mM TRIS/MES $(\mathrm{pH}=5.5)$. Then the protoplasts were filtered with medium I $\left(0.5 \mathrm{M}\right.$ sorbitol; $1 \mathrm{mM} \mathrm{MgCl}_{2}$; $5 \mathrm{mM}$ TRIS/HEPES $(\mathrm{pH}=7.0))$. Wheat protoplasts were precipitated for $8 \mathrm{~min}$ at $500 \mathrm{rpm}$, resuspended in medium II ( $0.5 \mathrm{M}$ sucrose; $1 \mathrm{mM} \mathrm{MgCl}_{2} ; 5 \mathrm{mM}$ TRIS/HEPES ( $\left.\mathrm{pH}=7.0\right)$ ). Then $1 \mathrm{ml}$ of medium I and $1 \mathrm{ml}$ of medium III $(0.4 \mathrm{M}$ sucrose; $0.1 \mathrm{M}$ sorbitol; $1 \mathrm{mM}$ $\mathrm{MgCl}_{2} ; 5 \mathrm{mM}$ TRIS/HEPES $(\mathrm{pH}=7.0)$ ) were added to the suspension. The mixture was centrifuged for $5 \mathrm{~min}$ at $1500 \mathrm{rpm}$ (wheat) and $8 \mathrm{~min}$ at $500 \mathrm{rpm}$ (rice). The layer of detached protoplasts was removed from the gradient and resuspended for loading dye.

The protoplasts were loaded with a ROS-sensitive probe with 5-(6)-chloromethyl-2,7dichlorodihydrofluorescein diacetate $\left(\mathrm{CM}-\mathrm{H}_{2} \mathrm{DCFDA}\right.$, Molecular Probes) at a concentration of $25 \mu \mathrm{M}$ for $10 \mathrm{~min}$ [9]. Then the protoplasts were precipitated for $8 \mathrm{~min}$ at $500 \mathrm{rpm}$. The precipitate was collected and resuspended. The measurement of the fluorescence level was carried out on a laser confocal scanning microscope Leica TCS SP5 MP at the resource centre of Saint Petersburg State University "Development of Molecular and Cellular Technologies" (63 $\times$ magnification, water immersion). The wavelength of excitation is $495 \mathrm{~nm}$ (white laser, 17\% power, 2 frames/min), the wavelength of emission is 500-550 nm [9].

In situ hypoxia and reaeration conditions were created in the POC-R2 Cell Cultivation System (PeCon). A thin glass slide coated with $0.1 \%$ polylysine was placed at the base of this chamber. The suspension of protoplasts $(50 \mu \mathrm{L})$ was applied to the glass. The incubation medium included $0.5 \mathrm{M}$ sorbitol, $0.05 \% \mathrm{PVP}, 0.2 \% \mathrm{BSA}$, and $1 \mathrm{mM} \mathrm{CaCl}_{2}$ in 5 $\mathrm{mM}$ TRIS/HEPES ( $\mathrm{pH} 7.0)$. The medium was degassed in vacuum $(-70 \mathrm{kPa})$ for $15 \mathrm{~min}$. Then the medium was saturated with either air (reaeration) or Nitrogen gas $\left(\mathrm{N}_{2}\right)$ to a final $\mathrm{O}_{2}$ concentration of $26-30 \mathrm{nmol} / \mathrm{ml}$ (severe hypoxia) for $45-60 \mathrm{~min}$. To create hypoxic 
conditions, protoplasts were poured in $2 \mathrm{ml}$ of the above-mentioned medium, the chamber was hermetically closed, and the measurements were carried out for $30 \mathrm{~min}$. To create conditions for in situ reaeration, the protoplast suspension was placed in $2 \mathrm{ml}$ of a hypoxic medium for 1 hour. Then the protoplasts were quickly transferred into glass, and $2 \mathrm{ml}$ of the aerated medium was added. The measurements were carried out for $30 \mathrm{~min}$ in the hermetically closed chamber [10]. The image files obtained in the experiment were processed using the Fiji program. All the experiments were carried out in at least 7 biological replicates. The statistical significance of differences in experimental data was assessed using the The Mann-Whitney U test.

\section{Results}

It is widely believed that $\mathrm{H}_{2} \mathrm{O}_{2}$ is involved in the transduction of the anaerobic signal into the cell of various higher plants [11]. However, the rapid accumulation of this ROS is observed only in the first seconds or minutes of the action of hypoxia (anoxia) conditions on plant tissues [3]. Our experiments with protoplasts of wheat, sensitive to anoxia [10] and rice, which is characterized by a fairly high resistance to lack of aeration [12], do not refute this point of view. An indirect assessment of the $\mathrm{H}_{2} \mathrm{O}_{2}$ content in protoplasts was carried out by analyzing the fluorescence of $\mathrm{CM}-\mathrm{H}_{2} \mathrm{DCFDA}$. The results obtained clearly demonstrated the rapid accumulation of $\mathrm{H}_{2} \mathrm{O}_{2}$, its concentration reached a maximum after 30 minutes of exposure of protoplasts of both plant species under hypoxic conditions (Fig. $1)$.

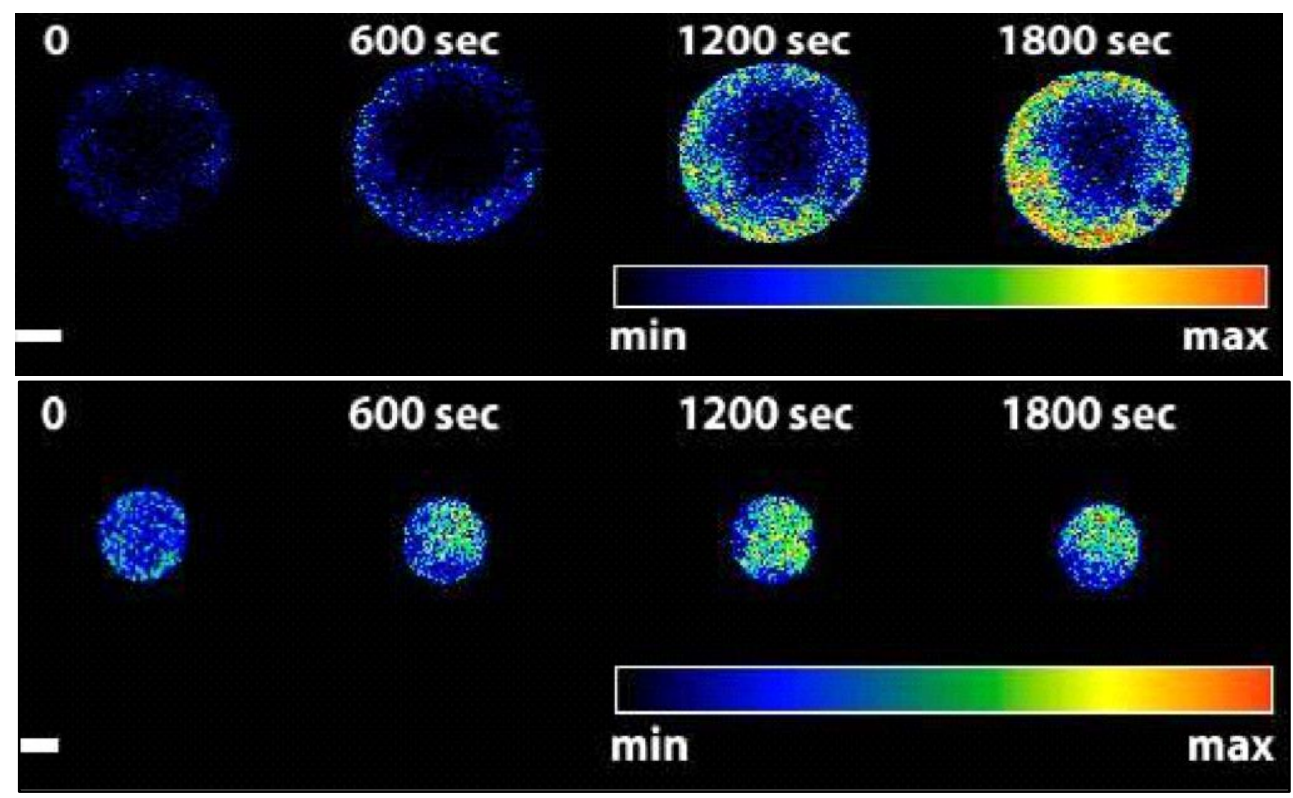

Fig. 1. ROS-dependent fluorescence of CM- $\mathrm{H}_{2} \mathrm{DCFDA}$ under the effect of hypoxia in wheat (A) and rice (B) protoplasts. The colour scale reflects the intensity of the fluorescence. The scale bar corresponds to 20 microns.

At the same time, the dynamics of $\mathrm{H}_{2} \mathrm{O}_{2}$ accumulation had some differences. Thus, the fluorescence intensity in rice protoplasts increased in direct proportion to the duration of hypoxia. A similar dependence for wheat protoplasts had "dips" when the fluorescence intensity first slightly decreased and then increased (Fig. 2). 


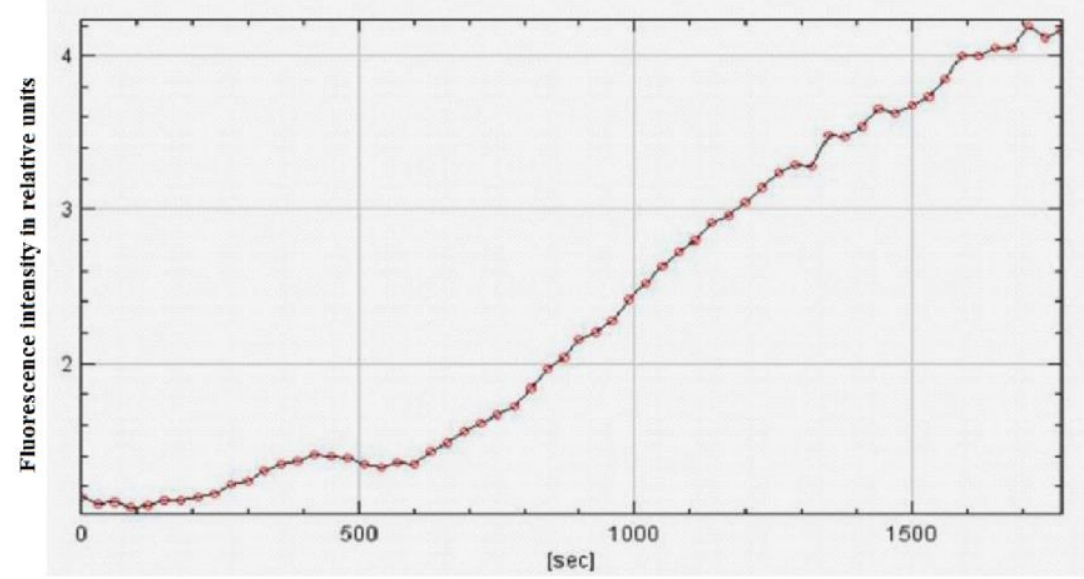

Fig. 2. ROS-dependent fluorescence of $\mathrm{CM}-\mathrm{H}_{2} \mathrm{DCFDA}$ in wheat protoplasts under hypoxic conditions $(\mathrm{P}<0.05)$.

Assessment of the intensity of $\mathrm{H}_{2} \mathrm{O}_{2}$ formation in protoplasts of higher plants under conditions of reaeration has not been previously performed by other authors. Nevertheless, this approach made it possible to analyze indirectly the degree of ROS involvement into the processes of the hypoxia (anoxia) signal transduction, to differentiate it against the background of ROS-dependent mechanisms of plant cell damage during oxygen deficiency (hypoxia and anoxia). Experimental data indicated that the effect of reaeration led to a Ushaped change of ROS-dependent $\mathrm{CM}-\mathrm{H}_{2}$ DCFDA fluorescence in protoplasts of both studied species. Nevertheless, the fluorescence intensity observed in rice protoplasts was more than 2 times higher than that for wheat protoplasts. Comparative analysis of ROS production under conditions of hypoxia and reaeration for protoplasts of each studied species is shown in Fig. 3.

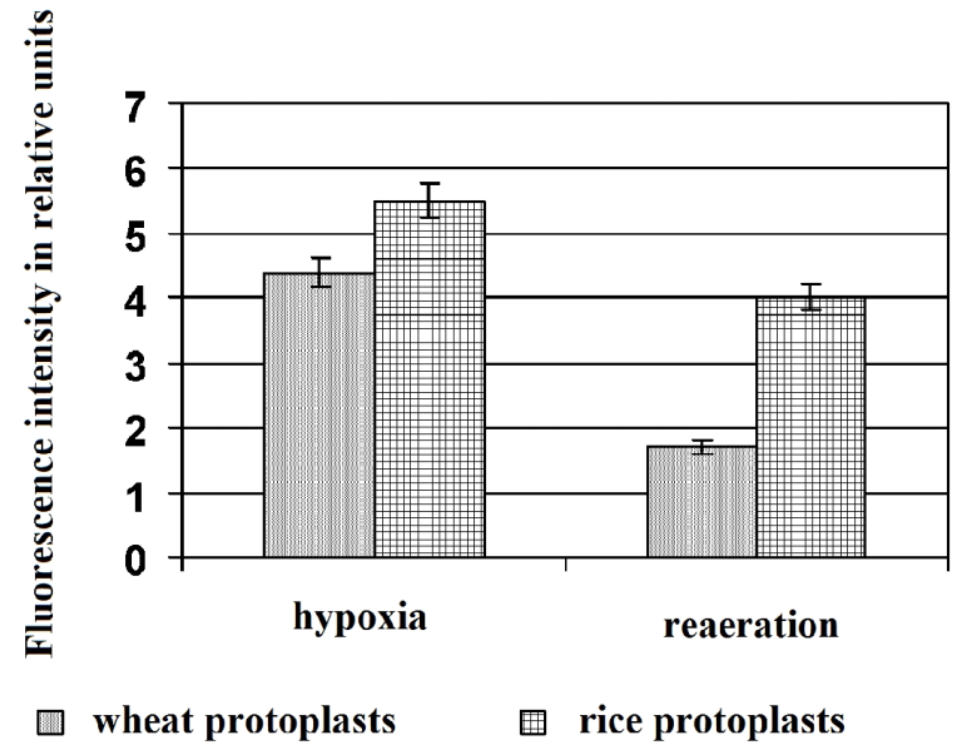

Fig. 3. Comparative analysis of ROS production under conditions of hypoxia and reaeration for protoplasts of studied species. 
It was found that the intensity of ROS-dependent fluorescence of CM- $\mathrm{H}_{2} \mathrm{DCFDA}$ in rice protoplasts exceeded those for wheat protoplasts. The greatest difference (more than 2 times) was characteristic for the conditions of reaeration. This result probably reflects greater resistance of rice to hypoxia. It is noteworthy that the intensity of $\mathrm{H}_{2} \mathrm{O}_{2}$ formation by protoplasts of both studied plant species during 30 min of hypoxia was higher than during short-term reaeration (after 1 hour of hypoxia). This indirectly indicates the possible participation of ROS in the processes of intracellular signal transduction under the conditions of oxygen deprivation.

\section{Discussion}

Higher plants often experience a lack of soil aeration, which occurs due to excess water during seasonal or perennial floods, as well as after heavy rains - the result of global warming of the Earth. Climate change in Russia is accompanied by natural phenomena (floods, avalanches, mudflows, hurricanes, sudden changes in weather), which complicate the work of enterprises in the agricultural sector [13]. Decrease of the oxygen content in the soil leads to a thinning of crops, a reduction in plant productivity [2]. Often the crops that form the basis of the Russian grain economy are exposed to low-oxygen conditions. This has a negative impact on economic activities of the main regions for the most winter-hardy wheat cultivars (The Southern Urals, Western Siberia, Volga region). According to the available literature data, traditional agricultural wheat varieties are sensitive to the level of soil aeration and quickly die in partial (hypoxia) or complete flooding (anoxia) [14]. At the same time, rice is known as the only crop that is resistant to the complete absence of oxygen (severe anoxia) [9, 15]. Therefore, the mechanisms of rice adaptation to oxygen deficiency are of great interest to the development of methods for selecting new agricultural plant varieties suitable for growing in conditions of flooding and waterlogging of soils in the middle zone of the Russian Federation.

It is widely believed that the main damaging effect on biopolymers of plant tissues is exerted not so much by oxygen starvation as by the process of restoring normal aeration conditions (so-called reaeration). In this case, plant cells are exposed to post-anoxic oxidative stress, coupled with the active formation of various ROS, which quickly transform into each other [9]. Among other forms of ROS, $\mathrm{H}_{2} \mathrm{O}_{2}$ is distinguished by a lower cytotoxicity, a long half-life, as well as the ability to penetrate cell membranes due to the facilitated diffusion mechanism [5, 12]. These features explain well the interest in the analysis of $\mathrm{H}_{2} \mathrm{O}_{2}$ in higher plants to determine the contribution of ROS to the transduction of hypoxia signals. Our experiments indirectly confirmed the functioning of such a mechanism. In the protoplasts of wheat and rice, a clear tendency towards a decrease of $\mathrm{H}_{2} \mathrm{O}_{2}$ concentration under reaeration conditions compared to hypoxia conditions was revealed. The intensity of $\mathrm{H}_{2} \mathrm{O}_{2}$ formation correlates well with the literature data on the degree of resistance of the studied plants to oxygen deficiency. However, the nature of the dependence of the $\mathrm{H}_{2} \mathrm{O}_{2}$ intracellular concentration on the duration of hypoxia exposure revealed some differences. Thus, for rice protoplasts, it obeyed a directly proportional relationship, while for wheat protoplasts it contained hyperbole elements (see Fig. 2). This may indicate the role ambiguity of ROS in the initiation of oxidative stress, as well as the induction of adaptive physiological and biochemical reactions of cells and tissues.

It is known that ROS are generated in a plant cell by both enzymatic and non-enzymatic methods. Their production under hypoxia (anoxia) conditions is provided by NADPH oxidases of the plasma membrane [16]. The typical representatives of this group are RBOH transmembrane proteins (Respiratory Burst Oxidase Homologs). The activation of RBOH under hypoxia conditions stimulates a sharp surge in the ROS production (the so-called first 
wave), which are able to transduce a signal to various tissues of Arabidopsis thaliana [17]. The second wave of ROS is generated by various intracellular compartments (chloroplasts, mitochondria, peroxisomes). Such ROS are involved in the transmission of retrograde signals to the nucleus and affect the transcription of genes for adaptation to stress factors. Change in the expression of these genes activates ATP synthesis pathways alternative to oxidative phosphorylation $[18,2]$. According to literary sources, the tolerance of higher plants to anoxia is determined by the slower oxidation of sugars - the main substrates for the synthesis of ATP. This makes possible flexible intracellular regulation of metabolic flows with complete or partial oxygen deficiency $[19,20]$. Therefore, the study of the energy metabolism characteristics of wheat cells sensitive to hypoxia, as well as the detection of signals interaction generated by ROS with other intracellular mediators, become important prerequisites for the design of flood-resistant varieties that are of great national economic importance for the Russian Federation [21].

\section{Conclusion}

The hypoxia (anoxia) conditions induce the formation of various ROS in the cells of higher plants. These molecules are involved in the transmission of stress signals to other cells of a multicellular organism. The biological role of ROS is accompanied not only by the disruption of the structure and functions of biopolymers (lipids, proteins, nucleic acids), but also by the activation of specific nuclear genes that determine adaptive modifications to oxygen deficiency. An indispensable condition for signal transduction during anoxic stress is a rapid change in the amount of ROS. The dependence of the $\mathrm{H}_{2} \mathrm{O}_{2}$ concentration (one of the forms of ROS) in the protoplasts of rice resistant to oxygen deficiency on the duration of hypoxia was directly proportional. For wheat protoplasts (sensitive to oxygen deficiency), a similar relationship was more complex. This indicates the ambiguous role of ROS in the initiation of oxidative stress, as well as in the induction of responsive adaptive physiological and biochemical reactions. Nevertheless, the mechanism of hypoxia signal transduction, as well as the degree of participation of other intracellular messenger molecules in its work, is not clear. To solve this problem, additional studies of the energy metabolism characteristics and general pathways of sugar catabolism in plant cells sensitive to oxygen deficiency are required. Such data will help to reveal the molecular basis of adaptation of higher plants to hypoxia (anoxia) conditions, to determine the strategy for the design of new wheat varieties resistant to flooding and waterlogging of soils in the middle zone of the Russian Federation.

\section{References}

1. O.A. Zhuykova, P.D. Smirnov, V.V. Yemelyanov, O.I. Bolotnikova, Materials of the scientific conference "Biotechnology and food technologies" dedicated to the 45th anniversary of biotechnological education in Russia (2020)

2. S.S. Medvedev, Plant physiology (2012)

3. M.F. Shishova, Reception and signal transduction in plants (2008)

4. A.R. Garifzyanov, Formation and physiological reactions of reactive oxygen species in plant cells, 2 (2011)

5. Y.E. Kolupaev, Ukrainian Biochemical Journal, 4 (2014)

6. H. Sies, D.P. Jones, Nature Reviews Molecular Cell Biology, 21 (2020)

7. H. Huang, F. Ullah, D. X. Zhou, M. Yi, Y. Zhao, Frontiers in Plant Science (2019) 
8. M.M. Zlotina, V.V. Yemelyanov, T.V. Chirkova, Vestniks of Saint Petersburg University, 2 (2011)

9. S.S. Gill, N. Tuteja, Plant Physiol. Biochem, 48 (2010)

10. V.V. Yemelyanov, M.F.Shishova, T.V. Chirkova, Lindberg S.M., Springer-Verlag (2011)

11. P. Sharma, A.B. Jha, R.S. Dubey, M. Pessarakli, J. Bot. (2012)

12. V.V. Yemelyanov, M.F. Shishova, Springer-Verlag (2012)

13. V.V. Oganesyan, Hydrometeorological research and forecasts, 373 (2019)

14. E.V. Ageeva, I.N. Leonova, I.E. Likhenko, Vavilovskii Zhurnal Genet Selektsii, 24, 4 (2020)

15. E. Solórzano, J. Francisco, S. González-Gordo, José M. Palma, Agronomy, 10, 7 (2020)

16. Y. Kou, J. Qiu, Z. Tao, International Journal of Molecular Science, 20, 5 (2019)

17. T.V. Chirkova, V.V. Yemelyanov, Biological communications, 63, 1 (2018)

18. Y. E. Kolupaev, Y.V. Karpets, Physiology and Biochemistry of the Cult. plants, 2 (2009)

19. X.X. Cheng, M. Yu, N. Zhang, Z.Q. Zhou, Q.T. Xu, F.Z. L.H. Mei, Qu, Protoplasma, 253, 2 (2016)

20. A.E. Shikov, T.V. Chirkova, V.V. Yemelyanov, Plant Physiology, 67, 1 (2020)

21. M.J. Moloi, A.J. van der Westhuizen, Journal of Plant Physiology, 16, 11 (2005) 